\title{
Integrating Spatial Data Infrastructures (SDIs) with Volunteered Geographic Information (VGI) for creating a Global GIS platform
}

\author{
Demetris Demetriou ${ }^{\star, \dagger}$, Michele Campagna ${ }^{\ddagger}$, \\ Ivana Racetin ${ }^{\S}$, Milan Konecny
}

*Public Works Department, 165 Strovolos Avenue, 2048 Nicosia, Cyprus

${ }^{\dagger}$ School of Geography, University of Leeds, LS2 9JT, Leeds, UK, demdeme@cytanet.com.cy

${ }^{*}$ DICAAR, Università Di Cagliari, Via Marengo 2, 09123 Cagliari, Italy ${ }^{\varsigma}$ Faculty of Civil Engineering, Architecture and Geodesy, University of Split, Matice hrvatske 15, 21000 Split, Croatia

'Department of Geography, Faculty of Science, Masaryk University, Kotlarska 2, 61137 Brno, Czech Republic

\begin{abstract}
Spatial Data Infrastructures (SDIs) are a special category of data hubs that involve technological and human resources and follow well defined legal and technical procedures to collect, store, manage and distribute spatial data. INSPIRE is the EU's authoritative SDI in which each Member State provides access to their spatial data across a wide spectrum of data themes to support policy-making. In contrast, Volunteered Geographic Information (VGI) is one type of user-generated geographic information (GI) where volunteers use the
\end{abstract}

How to cite this book chapter:

Demetriou, D, Campagna, M, Racetin, I, Konecny, M. 2017. Integrating Spatial Data Infrastructures (SDIs) with Volunteered Geographic Information (VGI) for creating a Global GIS platform. In: Foody, G, See, L, Fritz, S, Mooney, P, OlteanuRaimond, A-M, Fonte, C C and Antoniou, V. (eds.) Mapping and the Citizen Sensor. Pp. 273-297. London: Ubiquity Press. DOI: https://doi.org/10.5334/bbf.l. License: CC-BY 4.0 
web and mobile devices to create, assemble and disseminate spatial information. There are similarities and differences between SDIs and VGI, as well as advantages and disadvantages to both. Thus, the integration of these two data sources will enhance what is offered to end users to facilitate decision-making. This idea of integration is in its early stages, because several key issues need to be considered and resolved first. Therefore, this chapter discusses the challenges of integrating VGI with INSPIRE and outlines a generic framework for a global integrated GIS platform, similar in concept to Digital Earth and Virtual Geographic Environments (VGEs), as a realistic scenario for advancements in the short term.

\section{Keywords}

SDIs, INSPIRE, VGI, Global Integrated GIS platform

\section{Introduction}

Data hubs have arisen through the evolution of information technology, and aim to provide a centralised, unified data source that can be easily accessed by certain groups of users, or more widely by the public, to support a diversity of professional and/or other needs (Mangano, 2013). A special category of data hub is that of Spatial Data Infrastructures (SDIs; Williamson et al., 2003), which emerged during the mid-1990s (Delaney and Pettit, 2014). SDIs involve technological and human resources that follow well defined legal and technical procedures to collect, store, manage and distribute spatial data. On 14 March 2007, the European Parliament and Council adopted a Directive establishing the Infrastructure for Spatial Information in the European Community (INSPIRE) European SDI (European Commission, 2007). Following the INSPIRE Directive, Public Authorities (PAs) in each Member State should provide access to their SDI across a wide spectrum of data themes through a community geoportal, aiming thus to support policy-making and activities aimed at, but not limited to, the protection of the environment.

Whilst INSPIRE tries to unite and standardise existing Authoritative Geographic Information (AGI) made available by PAs in EU Member States, technologies that enable User-Generated Content (UGC) have also appeared (Moens et al., 2014) in web-based platforms (e.g. blogs, wikis, discussion forums, posts, chats, tweets), mobile computing and GPS devices. Hence, users have started to create and share data and information. Volunteered Geographic Information (VGI) is one type of user generated GI (Goodchild, 2007), where volunteers use the web and mobile devices to create, assemble and disseminate spatial information. Among the most well known VGI platforms are OpenStreetMap (OSM; Demetriou, 2016) and Wikimapia, but there 
are many others, covering a range of fields such as conservation, planning, and crisis management. Thus, there is a potential for VGI to become an important source of information that could benefit INSPIRE and similar projects and efforts; on the other hand, VGI could also benefit from INSPIRE through integration with official and reliable data and the need to adopt more strict specifications.

Although INSPIRE ${ }^{1}$ is a well organised, official and reliable platform that is based on strict standards, it provides data that are mainly used by experts and involves static information (with a limited level of detail in some cases) that is not updated very regularly because of the high costs involved. VGI, on the other hand, is captured unofficially by volunteers, often using cheap devices, e.g. a handheld GPS or smartphones; hence the data quality is usually limited and the data collection is not based on strict standards. However, real-time data can be collected anywhere by anybody, opening up concrete possibilities for data to be updated very regularly at little or no cost. Therefore, the integration of both types of data (Craglia, 2007; Budhathoki et al., 2008; Craglia et al., 2008; McDougall, 2009; Parker et al., 2012; Massa and Campagna, 2016) could potentially enhance what is delivered to end users, supporting the full spectrum of related needs, both professional, e.g. planning and spatial decision-making, and of the daily activities of citizens.

The idea of integration of VGI and authoritative data has arisen recently and been emphasised by several researchers (Budhathoki et al., 2008; Craglia et al., 2008; McDougall, 2009; Parker et al., 2012). In addition, the benefits of integration refer to both the organisations involved, i.e. National Mapping Agencies (NMAs; Olteanu-Raimond et al., 2017) that operate national INSPIRE geoportals, and those who run VGI initiatives, as well the end users. Although some efforts towards this integration have already been made (Craglia, 2007; Wiemann and Bernard, 2014), the literature suggests that this endeavour is in its early stages because several critical issues need to be considered and resolved. As a result, the available literature is limited and focuses on specific projects or technical issues (Botshelo, 2009) without attempting to investigate the broader picture of integration or setting out a conceptual framework. Further to this integration, the vision is the development of a global integrated GIS platform, which extends the capabilities of a typical data hub and the benefits of integration of SDIs with VGI by embedding on-line geospatial tools, to deliver both static and dynamic outputs to support planning and decision-making. Such visionary and/ or applied advanced geospatial tools and frameworks moving in this direction are the GeoWeb (Dangermond, 2005), Digital Earth (Craglia et al., 2008) and Virtual Geographic Environments (VGEs; Lin et al., 2013).

Based on the above, this chapter aims to discuss the challenges of integrating VGI with INSPIRE, and to outline a generic framework for a global integrated GIS platform, similar in concept to Digital Earth and VGEs, as a realistic scenario for advancements in the future. The remainder of this chapter is organised as follows: Section 2 provides an overview of SDIs and VGI, contrasting 
these two sources of data. This is followed by a discussion about critical issues that arise in INSPIRE and VGI integration (Section 3). In Section 4, the prospects of integration are examined, with some examples. Section 5 then presents an outline of a conceptual framework for an ideal global integrated GIS platform, while conclusions are summarised in Section 6.

\section{Spatial Data Infrastructures (SDIs) and Volunteered Geographic Information (VGI)}

Before discussing the various issues of integration between SDIs and VGI, an overview of each infrastructure and a comparison are presented, providing the necessary background.

\subsection{Spatial Data Infrastructures (SDIs)}

Data hubs are defined as community-run catalogues of useful, online datasets, which store a copy of the data or host them in a database and provide some basic visualisation tools (Open Knowledge Foundation, 2013). A typical data hub consists of four basic elements, as shown in Figure 1: Data, a Facilitator, a Custodian and End Users, which together form a dynamic communication cycle (Delaney and Pettit, 2014).

In particular, the Facilitator should provide a connection between the Custodian, i.e. the data hub's administrator, and the End Users; negotiate with the

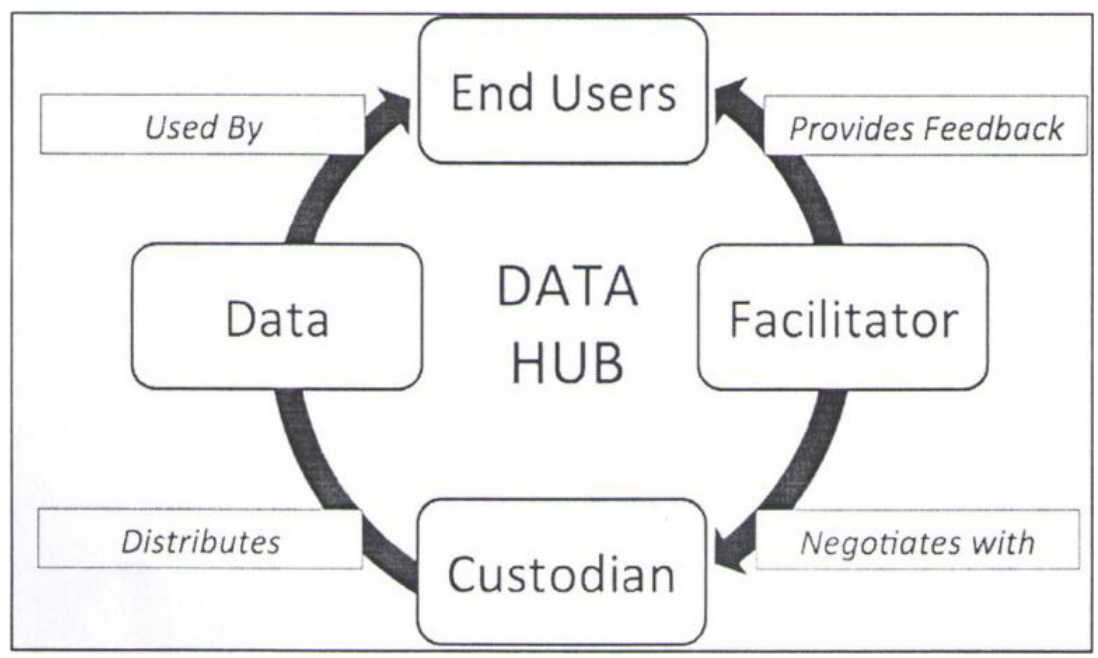

Fig. 1: Data hub conceptual communication - feedback cycle (adapted from Delaney and Pettit, 2014). 
Custodian in terms of the needs or problems; and provide feedback to end users. The role of the Custodian is to provide and distribute data, which will be used by the End Users. It is to be noted that the terms 'end users' and 'users', as used in this chapter, have a slightly different meaning: namely, while 'end users' utilise the data provided by the hub, they do not necessarily contribute to the development of the hub voluntarily, i.e. by delivering new data, updating existing data or sharing data - tasks that are carried out by 'users'. Obviously, 'users' can also be 'end users'; that is, they can have a double role.

Access to data hubs can be free and/or licensed. A data hub allows users to access, search and use a variety of data with associated metadata provided as a discrete set of formats. The data hub concept has been realised in many locations and contexts globally. Many scientific fields have collaborated to create research-specific data hubs to store and discover data and to distribute them to other researchers (Delaney and Pettit, 2014).

SDIs are a special category of data hubs (Williamson et al., 2003) that involve a framework of interacting elements, aiming to acquire, store, preserve, process, distribute, use and maintain data with 'a direct or indirect reference to a specific location or geographical area' (European Commission, 2007). The main elements of this framework are: spatial datasets and their metadata; networks services and technologies; standards that define the quality of the data; policies for distributing and managing the data; human resources; and a mechanism for coordinating and monitoring the whole infrastructure (European Commission, 2007; Iliffe, 2012). An SDI may be developed by national public bodies to support all of the spatially relevant activities in a country. Each national, regional or local SDI, as a node of INSPIRE, recognises the significance of metadata by ensuring all contributed data align to a minimum standard and aims to deliver up-to-date data and information to other government agencies and the general public (Steven, 2005) to support effective decision-making. Several SDIs have been developed (Craglia, 2007), e.g. the National Spatial Data Infrastructure (NSDI) in the United States in 1994 and INSPIRE in Europe.

\subsection{Volunteered Geographic Information (VGI)}

UGC is divided into two main types: non-georeferenced and georeferenced, as illustrated in Figure 2. The most popular forms of the former type include text messaging, social media interactions, photos, videos, blog entries, etc. Georeferenced UGC involves various forms of location-based technologies, such as location-based services (LBSs), location-based social networks (LBSNs), social network location sharing (SNLS), location-based games (LBGs) and locationbased social network games (LBSNGs; Odobašić et al., 2013). In particular, the LBS industry has profited from UGC primarily because ubiquitous and affordable smartphones equipped with multiple sensors foster geographic data collection. Similarly, LBSN leverage the power and high adoption rate of modern 


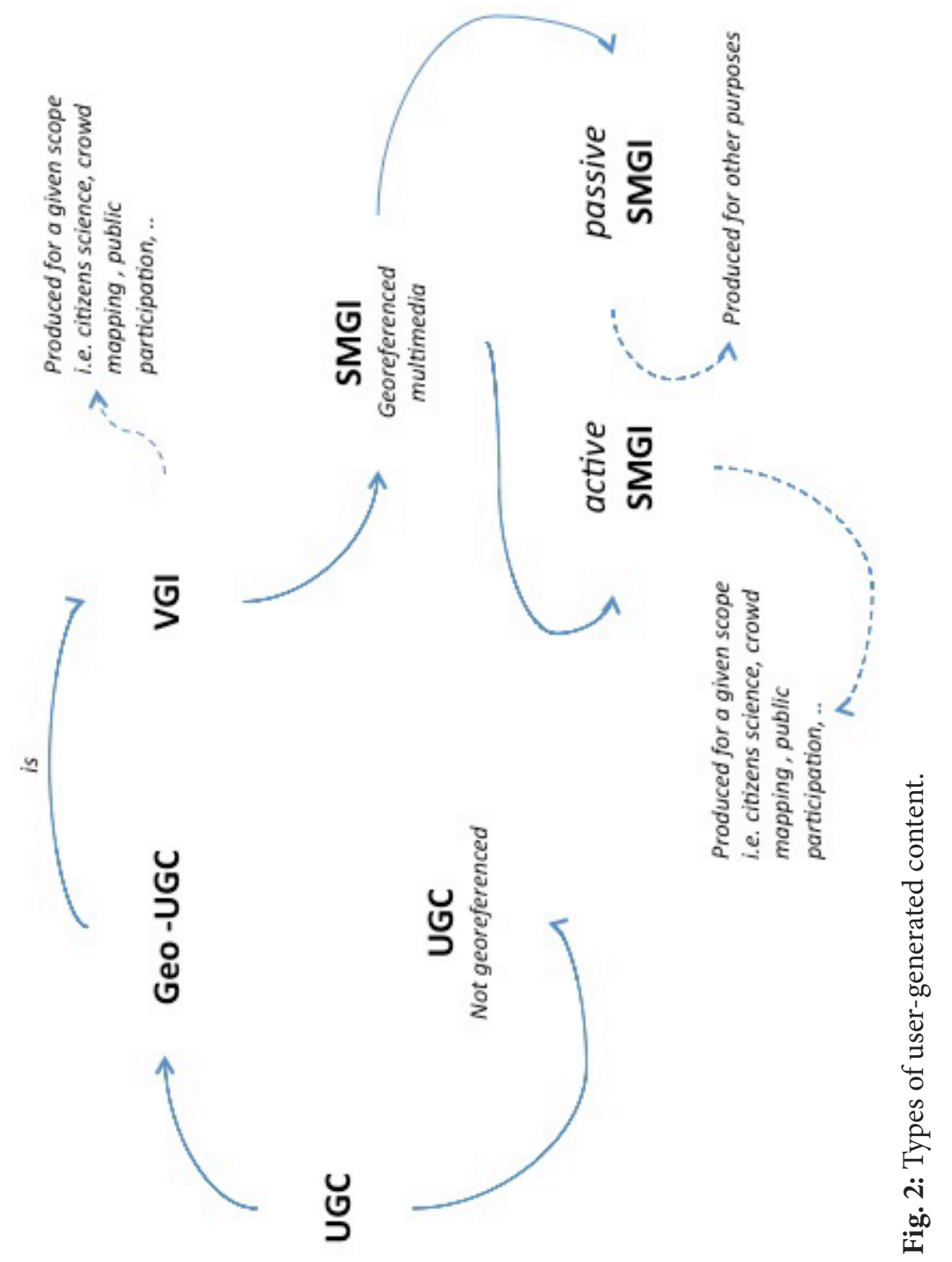


mobile devices to provide applications and services that allow users to share and discuss the real-world places they visit, as a part of their virtual interactions (Furey et al., 2013). In terms of social networks, location sharing has changed from a purpose-driven to a social-driven activity. Users traditionally shared their location with one other person (one-to-one) or with a small group (oneto-few); social networks, depending on the privacy/user settings, enable users to share their location with a large group (one-to-many) or with everyone (oneto-all; Tang et al., 2010). LBGs are games in which the game play somehow evolves and progresses based on a player's location. Thus, LBGs almost always support some kind of georeferencing technology, for example by using, WiFi, Near Field Communication, Bluetooth and satellite positioning such as GPS. The blend of LBGs and LBSNs creates LBSNGs, which are exemplified by a service like Foursquare.

Among the most popular geo-UGC-based technologies is VGI (Goodchild, 2007), or crowdsourced GI, which has arisen since 2007. VGI involves harnessing tools to create, assemble and disseminate geographic data provided voluntarily by individuals, and it can be generated through geobrowsers or smartphone apps, making use of georeferencing or geocoding tools and techniques. Two widely popular VGI platforms are OSM (Haklay, 2010) and Wikimapia (Wikimapia, 2015), but there are many others, covering many kinds of fields, such as conservation, planning, and crisis management. A special class of VGI is Social Media Geographic Information (SMGI), which can generally be divided into active and passive type (Figure 2). The former type is produced for a given scope, e.g. citizen science, crowd mapping or public participation, and users (i.e. volunteer contributors) are fully aware of this, such as in the case of OSM or Wikimapia. In contrast, the latter is produced for other purposes (i.e. users share passively or share unvolunteered information for undefined purposes, such as in the case of social network interaction) and may be accessed independently at a later stage for reuse by third parties for a variety of disparate aims.

\subsection{A Comparison of SDIs and VGI}

There are similarities and differences between SDIs and VGI (Castelein et al., 2010) regarding data, as well as advantages and disadvantages, and these are outlined in Figure 3. In particular, data provided by SDIs are captured by well trained specialists who are employed by formal public or private organisations, and through well defined workflows, using state-of-the-art technology (Castelein et al., 2010); hence the SDI approach is an official, top-down approach involving high costs. On the other hand, VGI is captured unofficially by volunteer-citizens (classified by Coleman et al. (2009) into five categories), through smart phones/devices that provide GPS and Internet access or using other simple aids to take measurements; it is a bottom-up process with limited or no 
operational costs. Whilst the former data are generally free of charge or can be licensed through a fee, the latter are always provided for free. Moreover, SDIs have a data-centric scope as they mainly provide data used by experts through GIS portals, while VGI delivers information to a broader audience of mainly non-experts through user-friendly GI platforms.

In addition, SDIs involve static information provided periodically and in some cases with a limited level of detail, while VGI has both static and dynamic (real-time) information, since it can process real-time, spatiotemporal information, and can provide a much greater level of detail in some cases. This suggests that VGI could be a potentially complementary source to SDI in providing relevant real-time data related to physical catastrophes, crisis management situations or humanitarian missions. Furthermore, SDI provides certified data based on strict and professional international standards and specifications such as that provided by the Open Geospatial Consortium (OGC) and International Standardisation Organisation (ISO), while VGI is based on essential data standards that vary from platform to platform; most importantly, the quality of their data is unknown.

The above comparison also reveals two weaknesses of SDIs: the lack of capacity for real-time data to be collected anywhere by anybody and the lack of the flexibility of very regular data updates at low or no cost. Thus, a combination of both technologies will enhance what is offered to end users to facilitate decisionmaking, and the idea of integration has been discussed by several researchers (Budhathoki et al., 2008; Craglia et al., 2008; McDougall, 2009; Parker et al., 2012). However, this challenge will not be an easy one, because the institutional framework of the integration will be complex due to the different requirements and scope underlying each technology.

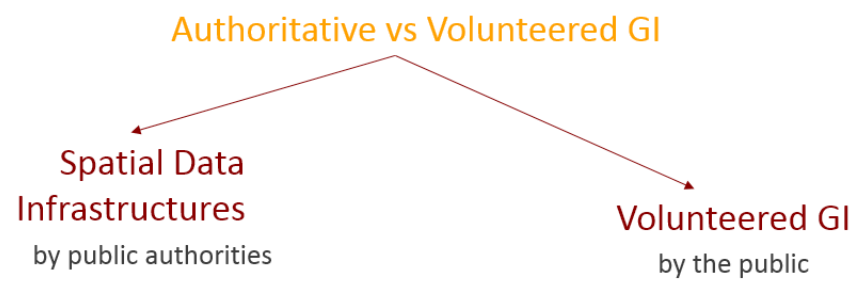

= Top-down

- Based on specifications

- Certified data

- Workflows

- Licensing

- Cost/benefits

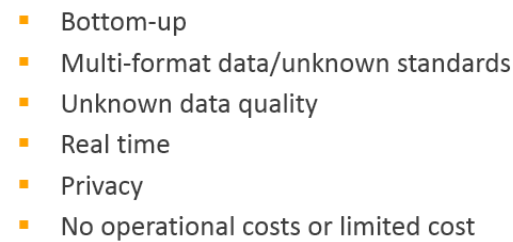

- Bottom-up

- Multi-format data/unknown standards

= Unknown data quality

- Real time

- No operational costs or limited cost

Fig. 3: The differences between SDIs and VGI. 


\section{Integrating VGI to INSPIRE}

The dominant European SDI is INSPIRE, and its integration with VGI is a difficult task because of several critical issues regarding the common implementing rules, which are discussed below. An overview of INSPIRE is first provided.

\subsection{The INSPIRE Directive}

INSPIRE, which has been defined by EU Directive 2007/2/EC (European Commission, 2007) and was adopted in 2007, establishes the requirement that each Member State should provide access to their SDI through a community geoportal operated by the European Commission or any other access point they wish to operate. The INSPIRE implementation provides a large-scale application of the open geoportal environment and is a big step forward in the development of an SDI in Europe. INSPIRE will overcome existing weaknesses and gaps in the interoperability of information resources across Europe by integrating them into a common framework (Craglia, 2007). The aim of INSPIRE is to assist policy-making and activities related to the environment and beyond; hence it involves data regarding a broad spectrum of fields, which are reflected in 34 spatial-data themes. The INSPIRE implementation represents a significant investment from all Member States, and has resulted in close to 300,000 spatial datasets being made available to the community through a standardised data-discovery site. The main INSPIRE portal allows users to search for datasets from across the EU from a single interface, and allows advanced search filters to be used to narrow down searches by geography, format or spatial theme. The INSPIRE portal only displays metadata for each dataset; it does not allow users to directly access any of the datasets, either manually or programmatically. However, each metadata resource contains a link to the data source, which may be a file, service or web application.

It should be noted that INSPIRE involves some general rules: it is based on existing SDI of Member States, and hence does not require the collection of new data, but demands the transformation of existing data to comply with its specification structure; and it does not affect intellectual property rights. In particular, the Directive also requires that common implementing rules be adopted in four main specific areas: metadata, data specifications, network services, and data and service sharing. These areas face critical integration issues, as discussed below.

\subsection{Critical Issues for Integration}

Following the INSPIRE Directive, Member States should provide metadata for spatial datasets/data series and/or for spatial data services. The metadata 
consist of 27 elements of information regarding the data resources, elements of information which are grouped into 10 categories: identification; classification; keywords; geographic location; temporal reference; quality and validity; conformity with the interoperability implementing rules; constraints related to access and use; organisation responsible for the resource; and metadata for metadata (European Commission, 2007). Clearly, populating all of these elements of metadata for VGI data will have a consequential time and cost. Furthermore, these elements cannot be gathered comprehensively by volunteers given current VGI practices. An issue is therefore who will be responsible for inputting all of these metadata and validating their reliability. Therefore, VGI metadata can be limited to only the basic information among the 27 elements provided by INSPIRE that can be input by the contributor, by the VGI system administrator or automatically by the system.

Similarly to metadata, the employment of common data specifications is a vital aspect of integration. Specifically, in order to ensure the interoperability of spatial information in INSPIRE, common international standards (those defined by ISO), technical specifications (e.g. regarding data types, code lists and enumerations, encoding, updating, the life cycle of spatial objects, reference temporal systems, and metadata) and minimum performance criteria for download services and transformation services have been defined (for each of the 34 related themes mentioned earlier). The issue of how to accommodate the diversified, dynamic and easy-to-access VGI data types to SDI is not a serious problem in technical terms; the problem is to define and apply minimum data requirements for VGI that are reasonable and achievable in order to satisfy data quality requirements (Wiemann and Bernard, 2014). Aspects of data quality such as positional accuracy, classification correctness and accuracy of the time measurement may follow the ISO 19157 standard (ISO, 2013; see Chapter 7 by Fonte et al. (2017) for more information on quality); a legally binding aspect is that of the topological consistency of the network data. VGI data quality and credibility vary from contributor to contributor (Flanagin and Metzger, 2008; Goodchild and Li, 2012; Foody et al., 2013); thus it is only up to a data provider whether they will respect data quality recommendations and whether they will report on recommendations in the metadata. Although some case studies on popular VGI platforms such as OSM have shown good and acceptable outcomes (Haklay, 2010), NMAs should evaluate the risks and problems that arise from the adoption of this new production system (Coleman et al., 2009; Bégin, 2012). Users should always be aware of how can they assess the credibility of data (Flanagin and Metzger, 2008) and contributors should be aware of the quality of the data used (Dassonville et al., 2003) and of whether they are fit for purpose. It is essential to develop tools that enable this evaluation. In addition, data quality can be improved by providing training on the needs of SDIs and on their protocols, and incentives can be awarded to contributors providing good work (see Chapter 5 by Fritz et al. (2017) for a discussion of incentives for volunteers). 
The interoperability of network services is also crucial for the joint operation of the systems. In particular, INSPIRE network services utilise one standard communication-protocol and binding technology for all service types to avoid mixing technologies: the Simple Object Access Protocol (SOAP), which ensures streamlined integration and implementation, as well as getting a maximum benefit from the offered services. SOAP is a protocol specification for exchanging structured information in the implementation of web services in computer networks. It uses the XML Information Set for its message format, and relies on other application layer protocols, most notably Hypertext Transfer Protocol (HTTP) or Simple Mail Transfer Protocol (SMTP), for message negotiation and transmission. In contrast to INSPIRE, it is reasonable that the various VGI platforms should use different communication-protocols and binding technologies through the platform owner's Application Programming Interfaces (APIs). However, VGI may reuse the two types of services provided by INSPIRE, i.e. viewing and downloading. The former operation is typically based on OGC Web Map Services (WMSs) or OGC Web Map Tile Services (WMTSs), which are easy to integrate into a VGI application from the technical as well as the legal point of view; the VGI application acts like a client application to a server, publishing data under the INSPIRE Directive. Most of the INSPIRE view services are provided free of charge, but there may be conditions that prevent their reuse for commercial purposes (European Commission, 2007). The latter type of service, download, is based on OGC Web Feature Services (WFSs), OGC Web Coverage Services (WCSs) and OGC Sensor Observation Services (SOSs), among others, which are also easy to integrate from a technological point of view. Data published through INSPIRE download services may also have associated fees, but these charges should not exceed the cost of collection, production, reproduction and dissemination, together with a reasonable return on investment (European Commission, 2007).

Once the aforementioned technical issues are resolved, an integrated data and service sharing policy should be defined. Currently, INSPIRE requires Member States to provide the institutions and bodies of the community with access to spatial datasets and data services in accordance with harmonised conditions based on a minimum set of conditions to be respected. Member States are permitted exceptions to data sharing, and can even completely restrict access to certain data or can set security measures for obtaining access to these datasets and data services; for example, public-data access that may threaten individual privacy or national security can be restricted. While SDI data are under the full control of each Member State and several data are provided free of charge, VGI data are generally freely accessible, even though in some cases access is limited through restrictions. However, inherently, VGI platforms encourage registration of new users not only in terms of access, but also in terms of inputting new data and editing existing data. As a result, some critical security aspects may arise for society. For instance, how can a criminal VGI contributor be identified if they try to promote illegal activities and fraudulent 
information? (Legal issues of VGI are discussed in Chapter 6 by Mooney et al., 2017.) The above discussion indicates that VGI cannot be ruled through a strict framework such as that applied for INSPIRE, because it involves volunteered pieces of many GI infrastructures without an authoritative structure and scope. Therefore, the focus should be on the minimum aspects that will ensure interoperability, credibility and security of services and data.

\section{The Prospects of Integration}

\subsection{Integration for Supporting Conventional Spatial Tasks}

The combination of INSPIRE and VGI provides great potential for creating a comprehensive information platform by linking the advantages of authoritative information, i.e. quality assurance and normative status, with VGI advantages, i.e. rapid, up-to-date and dynamic information (Wiemann and Bernard, 2014). As a result, this integration can benefit NMAs, administrators of VGI projects and end users, with consequent socio-economic impacts (Campagna and Craglia, 2012). In particular, NMAs may have a real opportunity to use crowdsourced data to update some of their databases when the update is not done by them regularly due to the high costs involved or to add new data that are not available to them (Coleman et al., 2009). They can also use crowdsourced data to detect changes or vernacular place names (Olteanu-Raimond et al., 2017). On the other hand, INSPIRE can serve as a basis for validating VGI information (Wiemann and Bernard, 2014). Furthermore, end users may use this mix of official and spatio-temporal data for any relevant purpose, i.e. for leisure (to walk in unexplored natural tracks), for receiving notifications about a fact (e.g. the impacts of an earthquake), for travelling (i.e. which travel route to follow) and for professional/ authoritative decision-making (e.g. how to manage a physical catastrophe or a crisis; Craglia, 2007; Wiemann and Bernard, 2014).

Some efforts towards VGI/SDI integration for the aforementioned purposes have already occurred (Craglia, 2007), e.g. the Linked Map project, which links GI from different sources, in particular SDI and VGI, through the paradigm of Linked Data (Lopez-Pellicer and Barrera, 2014). Linked Data connects related data through Web technologies. The Linked Map project has converted government datasets provided by the Spanish National Geographic Institute to Linked Data into Resource Description Framework (RDF) data, so that these datasets can be linked to VGI sources (OSM, DBpedia, etc.) and can be integrated using RDF links. RDF is a standard model for data interchange on the Web; RDF links enable Linked Data browsers and crawlers to navigate between data sources and to discover additional data. Another successful example is the case of the Ordnance Survey, which has linked an administrative geography dataset to other datasets on the Web, demonstrating the advantages of 
explicitly encoding topological relations between geographic entities over traditional spatial queries (Goodwin et al., 2008).

\subsection{Integration with Social Media}

Both active and passive Social Media Geographic Information (SMGI) can be integrated with SDIs in a GIS environment to perform qualitative and quantitative spatial, or more complex, multidimensional, analyses (Jankowski et al., 2010; Bugs, 2014; Campagna et al., 2015; Longley and Adnan, 2016). In particular, the integration of INSPIRE and VGI may generate a higher level of knowledge than INSPIRE alone, especially in those domains where the social component of data plays a relevant role, such as in politics, geo-marketing, tourism or spatial planning. The INSPIRE model may be extended through integration with SMGI, where multimedia data (i.e. texts, images, videos or audio) and user evaluations of the portrayed objects or phenomena are given with a time-stamp, enabling various kinds of new analysis, such as the spatial, temporal and statistical analysis of user interests and preferences; multimedia analyses; behavioural analyses; or combinations of these analyses, among others. Regarding the spatial analysis of user interests, the high number of georeferenced posts on social media platforms such as Twitter, Instagram, YouTube, Panoramio and Flickr can be used to investigate the patterns of user interests in space using density (Campagna, 2014) and clustering functions (Massa and Campagna, 2014). Data from such platforms can be accessed through APIs, georeferenced and saved as spatial data layers. Using SDI services such as WMSs or WFSs, GIS software can easily access the social media platform through the API, enabling the seamless integration of AGI and geo-UGC, as demonstrated by Massa and Campagna (2014). The overlay of spatial data layers with topographic SDIs such as administrative boundaries may offer useful hints to public authorities in understanding not only which places are important to the community and how they are perceived (Campagna, 2014), but also the composition of a community, e.g. local people, commuters, tourists or others.

Similarly, the temporal reference is often an available attribute in SMGI, which enables the study of when given places or infrastructures and services are used at different points in time. In addition, spatial statistics of user preferences, i.e. the collecting of posts by location, enables planners to analyse patterns in user interests at different scales. An example is given in Floris and Campagna (2014), where hotspot analysis has been used at the regional level to study tourist preferences by profile, before further analysing single hotspots with a tool embedded in ArcGIS called the Spatio-Temporal Textual analysis (Spatext-STTx) suite and with geographically weighted regression to explore, at the local level, what physical and locational factors may affect those preferences. Furthermore, multimedia analysis is well developed in the case of text analytics. However, it is currently more difficult to automatically extract useful 
information from images, video or audio. In the case of text, many software packages can be used to apply simple (i.e. calculating word frequency, or tag clouds) to more advanced (e.g. sentiment analysis) text analysis techniques. These techniques can be easily applied to subsets of SMGI obtained by spatial, temporal or user query. Moreover, user behavioural analysis, i.e. querying SMGI by a user, enables the study of user behaviour in space and time. This information can be used to analyse, for example, whether a public space is visited by local people or by outside visitors. This information may also be useful for profiling: for the users visiting a certain place or service, user spatiotemporal footprints can be defined to identify people who mainly move locally, regionally or internationally, and where they come from.

An additional application of the Spatext (STTx) suite is that made in a case study for the cyclone Cleopatra in Sardinia (Italy) to extract all relevant data and information (e.g. perceptions, opinions and needs from the local communities) from social media, i.e. Twitter, YouTube, Wikimapia and Instagram. These data were then integrated with the latest official datasets for further analysis and relevant action by decision-makers. Another related web application called 'Place, I care' was employed to support urban and regional planning processes. In particular, the aim was to collect information from concerned citizens about the physical, environmental and socio-cultural space to support collaborative and participatory planning. Although they have not been verified yet through a systematic analysis, there have been several case studies on the application of STTx in the same areas with different SMGI sources, where different types of users returned similar results, suggesting further research should be devoted to better understanding the issue of representativeness.

The above novel analytics may result not only in increasing the real-time monitoring capability of geo-UGC in representing the state of territorial systems, but also in supporting public participation and dialogue among digitallyenabled communities, which increasingly represent a substantial share of the total population in most countries. Other similar examples can be found in several domains. For example, the US Geological Survey (USGS) uses social networking to collect real-time, earthquake-related messages and early information to accelerate the delivery risk and response. Other related initiatives aim at (spatial) data collection, e.g. Project $\mathrm{Noah}^{2}$, which is a citizen science web/mobile tool developed to explore and document wildlife around the globe. Similarly, the ZmapujTo.cz mobile application ${ }^{3}$ was developed in 2012 in the context of an ecological project to combat illegal dumping grounds in the Czech Republic and contribute to solving this problem with the involvement of citizens and relevant authorities. At the time of creation, there was only a database of old ecological burdens, which covered the illegal dumps only marginally. In order to cover the largest possible area and utilise the potential of crowdsourced data, a platform was founded for information-gathering from citizens. The modern, efficient and widely-accepted platform was chosen for mapping while the mobile application and interactive web form were used for 
reporting. More than 2500 illegal dumps were reported, and more than 40 municipalities and towns took part during the lifetime of the first version. In March 2014, the second version of ZmapujTo.cz was launched. This version introduced several new features. The most important change was the ability to report not only illegal dumping, but also a variety of other problems that one can encounter both in town and in the countryside. The entire website was redesigned, including an interactive map for efficient, fast and intuitive work. Further to the aforementioned applications, many other initiatives are aimed at supporting pluralism and public participation in decision-making, such as in the case of the SoftGIS approach (Kahila and Kyttä, 2009) adopted in the design of the Maptionnaire web platform (Kahila-Tani et al., 2016).

While early experiences in SDI/VGI integration and analyses may still be limited to expert research laboratories or to the fortresses of the social media corporations, institutional initiatives such as MYGEOSS may trigger further development in this domain. MYGEOSS is an ongoing project (2015-16) of the European Commission to develop smart Internet applications based on the Global Earth Observation System of Systems (GEOSS) to inform European citizens about the changes affecting their local environment. Specifically, within this project, a number of interactive apps were developed that reuse official spatial data to offer interactive services to the end users. For example, an application called 'Know Your City!', developed by UbikGS, presents social, economic and environmental indicators on a map-based quiz. Similarly, 'Loss of the Night', created by Interactive Scape GmBH \& GFZ, is an application enabling citizen scientists all over the world to collect quantitative information on the changing nighttime environment, and MYGEOSS Phenology App Response was produced by the Friedrich-Schiller University to support vegetation phenology analysis using satellite data and data collected by citizens ${ }^{4}$.

Despite the aforementioned efforts, Lopez-Pellicer and Barrera (2014) note that the integration of INSPIRE with VGI has not gained the expected attention yet, and this especially from large producers of GI, because of the technical disadvantages of the current Linked Data mechanism (Schade et al., 2010). Similarly, Wiemann and Bernard (2014) state that this integration effort is in its early stages, because several critical issues, which have been discussed earlier, need to be considered. Therefore, it seems that there is still a long way ahead for a full integration and operation of a global GIS platform, which is a concept set out in the next section.

\section{Towards a Global Integrated GIS Platform}

During the last few decades, the world has evolved rapidly because of the continuous increase in the urban population, new needs, modern lifestyles and technological advancements, creating millions of individual activities with environmental, economic and social impacts at different levels. As a result, sus- 
tainability at various levels and contexts has been introduced as one of the core aims of society, sustainability which will be better met if we understand the complexity of interactions and interrelations between the parameters involved. This suggests the need for dynamic information systems that provide reliable, accurate and real-time data to support intelligent planning and management in order to reach optimum decisions. Visionary and/or applied advanced geospatial tools and frameworks that move in this direction, such as the GeoWeb (Dangermond, 2005), Digital Earth (Craglia et al., 2008) and VGEs (Lin et al., 2013), have been proposed.

The GeoWeb is a computer network providing the ability to integrate and share geospatial information locally or globally via the Internet. Through the GeoWeb, the ideal system would be a wide network of distributed GIS services constructed and implemented by various inter-organisational collaborative agreements so that individual systems and communities might use each other's services, splitting the world into geographic components and allowing the dynamic integration of knowledge. The communities involved may range from simple users to governments, business enterprises and professionals focusing on improving their decision-making. Gradually, these communities may expand, interoperate more and become increasingly synergistic; hence the system might be driven by the thousands to millions of participants currently using websites such as Google Earth and OSM. Eventually, these services could provide a global network of open-access geographic knowledge about the planet and online applications (open access and licence-based) for processing this information to produce the outputs for decision-making. These functionalities may support a whole range of applications and purposes, supporting regional, national and even global applications, solving issues ranging from routine, static and structured problems to problems that are complex and unstructured (including those demanding real-time responses) and that depend on cross-organisation and cross-discipline collaboration. Both GIS professionals and citizens sensors have a role in this system. The former have the skills, knowledge and experience of authoritative system development and operation, while the latter represent the 'VGI-soldiers' across space and time who voluntarily collect and share valuable static or real-time information not available to SDIs (Dangermond, 2015).

Similarly, the vision of Digital Earth as defined by Craglia et al. (2008), which refers to a virtual globe system, would provide access to vast amounts of spatiotemporal multi-geoinformation for various levels of users - including modelling tools to facilitate decision-making. Digital Earth has eight key characteristics: it has multiple connected globes/infrastructures addressing the needs of different audiences; it is problem-oriented, i.e. focused on various key application themes such as the environment, health and societal issues; it enables space-temporal search in real-time from both sensors and humans; it allows spatial-based queries and advanced spatial analysis; it provides access to models as well as to 'what if' scenarios and forecasts; it supports the visualisation of 
abstract concepts and data types regarding global social issues, e.g. low income and poor health; it is based on open access and public participation across multiple technological platforms and media; and it is engaging, to enhance interactive and exploratory learning for multidisciplinary education and science. Five use cases that would comprise the vision of Digital Earth involving a unique platform have been provided by Goodchild (2012). These use cases involve Digitial Earth as a geoportal, a visualisation service, a platform for simulation and prediction, a source of unprecedented spatial and temporal resolution, and a technology fully integrated into human activities.

In a similar vein, VGEs involve a new generation of Web-based virtual geographic analysis platforms to facilitate the advanced exploration of physical, environmental, socio-economic and other phenomena to solve related problems at a deeper level by combining state-of-the-art geotechnology and knowledge. Such a VGE system would consist of four basic components: (i) the data component for the integration, organisation and management of geographic information; (ii) the modelling and simulation component for the dynamic analysis of geographic phenomena by providing experts from various disciplines with an open access platform to develop and disseminate distributed advanced models in an easy and collaborative way; (iii) the interactive component between the system and users that includes external and internal data collection tools; and (iv) the collaborative component that enables group decisionmaking for significant societal problems through public participation in the processes carried out by experts.

Although the concept of Digital Earth, the existing technology of the GeoWeb and the use cases for VGEs have a common aim and functions, i.e. to provide advanced geodata hubs and sophisticated spatial analysis tools on the Web, they have some differences in terms of their focus. In particular, Digital Earth and VGEs involve extended capabilities beyond sharing knowledge and geoinformation such as the GeoWeb's, by providing advanced virtual reality, processing, simulation and analysis models for solving a wide range of complex spatial problems. In addition, VGEs involve more problem-oriented geotechnology tools that inherently have some of the features of planning and decision support systems, while the Digital Earth concept aims to provide more abstract tools for investigating the spatial interactions of certain domains.

Based on the aforementioned visions, we try to shift from a conceptual context for creating a new-generation geographic tool, to a more practical and tangible framework for developing a global integrated GIS platform, as illustrated in Figure 4. This framework extends the capabilities of a typical data hub and the benefits of integration of SDIs with VGI. In particular, the system consists of three main components: integrated data infrastructures, integrated online applications and a system for providing outputs (both static and dynamic) that could lead to decision-making and actions. As an alternative to providing wide access to a single source of data, the Integrated Data Infrastructures component can provide distributed data mashups by integrating vast stores of information 
(from many sources in the public and private sector as well as from citizens) and of many different types of data, along with geospatial services that can interact and be used to create new information. The data sources can be SDIs such as INSPIRE or NSDI in the United States, VGI platforms created through various projects (e.g. OSM and Wikipedia), social media (e.g. Facebook or Twitter) and other media such as emails, mobile phones, Instant messenger, etc. Existing services can be combined to make new services, and Geocommunities, which are currently fragmented, may be consolidated in a loosely coupled environment and create new synergies (Esri, 2006).

The integration of online applications could provide functionalities from simple publishing and mapping/visualisation to advanced GeoComputation modelling (Abrahart and See, 2014). In particular, the current Web-GIS services can be extended to provide not only easy map publishing and viewing through VREs, but also basic GIS functions, such as querying, buffering, overlays, etc., through Open Access (or licence-based) online GIS software. In addition, focused GIS applications, in the form of different thematic modules (i.e. for planning, transport, the environment, etc.) embedded in the online GIS, may be offered through distributed geo-services based on Web, GIS servertechnology and service-oriented architecture (SOA) that is open, interoperable, and dynamic, based on common data and service standards and specifications. Using the SOA model with GIS services, users can integrate their desktop and departmental solutions into implementations that connect many departments and organisations (Dangermond, 2008). The Web Services architecture allows users to both federate their distributed systems and integrate GIS and spatial processing with other IT business systems, such as Enterprise resource planning (ERP), Customer relationship management (CRM) and Supervisory control and data acquisition (SCADA). While this has been possible for some time, the advent of SOA and simple technologies to integrate these services has made it much easier and promises to greatly expand the GIS market. Ideally, in this context, easy-to-build ad-hoc advanced spatial models for GeoComputation that employ artificial intelligence techniques, for example, for solving complicated problems might be the biggest achievement of this system.

The results of the system could take the form of Dynamic Outputs. Outputs, which result from the processing of static or real-time information, can have any form, i.e. they can take the form of maps, reports and messages, and mass notification alerts. In particular, maps and reports in text or tabular form are the custom outputs of a GIS and can be used by users for decision-making and appropriate actions. Messages, e.g. through phone calls, emails, SMS, Viber etc., refer to real-time reporting to administrations and organisations. Similarly, mass notification alerts refer to broad notifications, or alerts, sent to people in a specific geographic region in emergency or crisis management situations. The tremendous high-speed evolution of the Web and Geospatial technologies suggests that this 'super' global Geo-system is not far away. 


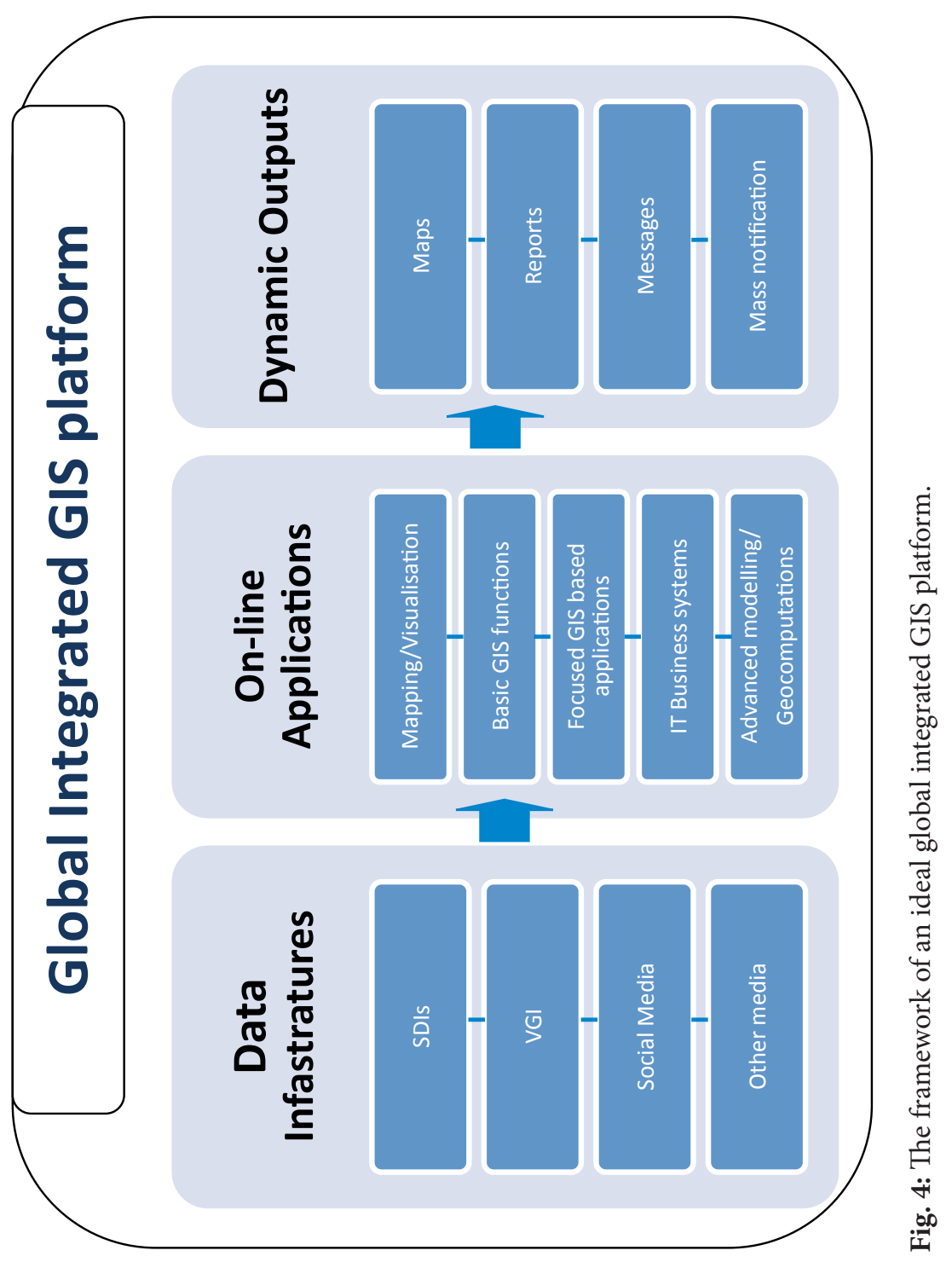




\section{Conclusions}

The integration of SDIs, and in particular INSPIRE, with VGI may potentially provide considerable benefits for all stakeholders involved, i.e. public and private organisations, professionals and citizens, because each technology may complement the other. In particular, benefits may include benefits for specific professional groups dealing with spatial problems; for planning and decisionmaking; and for the wider community, which may enable the dissemination and uptake of real-time updated information regarding daily activities (e.g. traffic incidents) or emergency situations, physical catastrophes or unknown threats. Although some early efforts towards this integration have been made, this project is not an easy task, since several technical and institutional issues need to be resolved, as discussed earlier. Ideally, the integration could be extended to creating a global integrated GIS platform, whose general framework has been presented and involves similar visions and concepts to Digital Earth and VGEs. The next steps should be focused on the establishment of a wider network of involved stakeholders, i.e. academia, industry, public authorities, citizens and NGOs, in the context of a well defined project (e.g. through a COST Action) to set up a robust framework that covers all of the aspects of the project, from the initial concept to its implementation, in order to achieve successful examples of integration and, ideally, an integrated GIS platform.

\section{Acknowledgements}

We would like to thank Dr Linda See, from the International Institute for Applied Systems Analysis (IIASA), Austria, and both internal and external reviewers for their valuable comments and suggestions on the original manuscript. We would also like to acknowledge the general support provided by the Chair of COST Action TD1202, Prof. Giles Foody, from the School of Geography, University of Nottingham, UK.

\section{Notes}

${ }^{1} \mathrm{http}: / /$ inspire.ec.europa.eu/

${ }^{2}$ http://www.projectnoah.org/

${ }^{3} \mathrm{http} / / / \mathrm{www} . Z \mathrm{Zmapuj}$ To.cz

${ }^{4} \mathrm{http} / / /$ digitalearthlab.jrc.ec.europa.eu/mygeoss/results.cfm

\section{Reference list}

Abrahart, R.J., See, L. (Eds.), 2014. GeoComputation, 2nd ed. CRC Press, Boca Raton, FL. 
Bégin, D., 2012. Towards integrating VGI and national mapping agency operations - A Canadian case study, in: Proceedings of the GIScience Conference, Columbus, OH, USA, 18-21 September 2012. Available at: http://web.ornl.gov/sci/gist/workshops/2012/documents/Begin,\%20 Daniel\%20-\%20Paper.pdf [Last accessed 16 May 2017].

Botshelo, S., 2009. Assessing Alternative Technologies for Use of Volunteered Geographic Information in Authoritative Databases. M.Sc.E. Thesis Department of Geodesy and Geomatics Engineering Technical Report No. 269. University of New Brunswick.

Budhathoki, N.R., Bruce, B. (Chip), Nedovic-Budic, Z., 2008. Reconceptualizing the role of the user of spatial data infrastructure. GeoJournal 72, 149160. DOI: https://doi.org/10.1007/s10708-008-9189-x

Bugs, G., 2014. Tecnologias da informação e comunicação, sistemas de informação geográfica e a participação pública no planejamento urbano. Tesi de dotourado. Universidade Federal do Rio Grande du Sul.

Campagna, M., 2014. The geographic turn in social media: Opportunities for spatial planning and Geodesign, in: Murgante, B., Misra, S., Rocha, A.M.A.C., Torre, C., Rocha, J.G., Falcão, M.I., Taniar, D., Apduhan, B.O., Gervasi, O. (Eds.), Computational Science and Its Applications - ICCSA 2014. Springer International Publishing, Cham, pp. 598-610.

Campagna, M., Craglia, M., 2012. The socioeconomic impact of the Spatial Data Infrastructure of Lombardy. Environment and Planning B: Planning and Design 39, 1069-1083. DOI: https://doi.org/10.1068/b38006

Campagna, M., Floris, R., Massa, P., Girsheva, A., Ivanov, K., 2015. The role of Social Media Geographic Information (SMGI) in spatial planning, in: Geertman, S., Ferreira, Jr, J.F., Goodspeed, R., Stillwell, J. (Eds.), Planning Support Systems and Smart Cities, Lecture Notes in Geoinformation and Cartography. Springer International Publishing, pp. 41-60.

Castelein, W., Grus, L., Crompvoets, J., Bregt, A.K., 2010. A characterization of VGI, in: Proceedings of the 13th AGILE International Conference on Geographic Information Science. Guimarães, Portugal, 10-14 June 2010. Available at: https://agile-online.org/conference_paper/cds/agile_2010/ shortpapers_pdf/106_doc.pdf [Last accessed 16 May 2017].

Coleman, D.J., Georgiadou, Y., Labonte, J., 2009. Volunteered geographic information: The nature and motivation of produsers. International Journal of Spatial Data Infrastructures Research 4, 332-358.

Craglia, M., 2007. Volunteered Geographic Information and Spatial Data Infrastructures: when do parallel lines converge? Position Paper for the NCGIA and Vespucci Workshop on Volunteered Geographic Information. Santa Barbara, CA, 13-14 Dec 2007. Available at: http://ncgia.ucsb.edu/projects/ vgi/docs/position/Craglia_paper.pdf [Last accessed 16 May 2017].

Craglia, M., Goodchild, M.F., Annoni, A., Camara, G., Gould, M., Kuhn, W., Mark, D., Masser, I., Maguire, D., Liang, S., Parsons, E., 2008. NextGeneration Digital Earth: A position paper from the Vespucci Initiative 
for the Advancement of Geographic Information Science. International Journal of Spatial Data Infrastructures Research 3, 146-167. DOI: https:// doi.org/10.2902/

Dangermond, J., 2015. Geospatial Technology and the Future of the City, ArcNews Online. Available at http://www.esri.com/esri-news/arcnews/ winter1415articles/geospatial-technology-and-the-future-of-the-city [Last accessed 1 May 2017].

Dangermond, J., 2008. GIS and the GeoWeb, ArcNews Online. Available at http://www.esri.com/news/arcnews/summer08articles/gis-and-geoweb. html [Last accessed 1 May 2017].

Dangermond, J., 2005. GIS Helping Manage our World, ArcNews Online. Available at http://www.esri.com/news/arcnews/fall05articles/gis-helpingmanage1of2.html [Last accessed 1 May 2017].

Dassonville, L., Vauglin, F., Jakobsson, A., Luzet, C., 2003. Quality management, data quality and users, metadata for geographical information, in: Shi, W., Fisher, P., Goodchild, M.F. (Eds.), Spatial Data Quality. CRC Press, pp. 214-227.

Delaney, P., Pettit, C., 2014. Urban Data Hubs Supporting Smart Cities, in: Winter, S., Winter, S., Rizos, C. (Eds.), Research@Locate 14. Canberra, Australia.

Demetriou, D., 2016. Uncertainty of OpenStreetMap (OSM) data for the road network in Cyprus, in: Proceedings of SPIE 9688, Fourth International Conference on Remote Sensing and Geoinformation of Environment (RSCy2016), 968806, Paphos, Cyprus, 4-8 April 2016. DOI: https://doi. org/10.1117/12.2239612

Environmental Systems Research Institute (Esri), 2006. The GeoWeb: A Vision for Supporting Collaboration, ArcNews Online. Available at http://www. esri.com/news/arcuser/0206/geoweb.html [Last accessed 1 May 2017].

European Commission, 2007. DIRECTIVE 2007/2/EC OF THE EUROPEAN PARLIAMENT AND OF THE COUNCIL: Establishing an Infrastructure for Spatial Information in the European Community (INSPIRE). Official Journal of the European Union.

Flanagin, A., Metzger, M., 2008. The credibility of volunteered geographic information. GeoJournal 72, 137-148.

Floris, R., Campagna, M., 2014. Social Media Geographic Information in tourism planning. Tema. Journal of Land Use, Mobility and Environment 0.

Fonte, C C, Antoniou, V, Bastin, L, Estima, J, Arsanjani, J J, Bayas, J-C L, See, L and Vatseva, R. 2017. Assessing VGI Data Quality. In: Foody, G, See, L, Fritz, S, Mooney, P, Olteanu-Raimond, A-M, Fonte, C C and Antoniou, V. (eds.) Mapping and the Citizen Sensor. Pp. 137-163. London: Ubiquity Press. DOI: https://doi.org/10.5334/bbf.g.

Foody, G., See, L., Fritz, S., Van der Velde, M., Perger, C., Schill, C., Boyd, D.S., 2013. Assessing the accuracy of volunteered geographic information arising from multiple contributors to an internet based collaborative project. Transactions in GIS 17, 847-860. DOI: https://doi.org/10.1111/tgis.12033 
Fritz, S, See, L and Brovelli, M. 2017. Motivating and Sustaining Participation in VGI. In: Foody, G, See, L, Fritz, S, Mooney, P, Olteanu-Raimond, A-M, Fonte, C C and Antoniou, V. (eds.) Mapping and the Citizen Sensor. Pp. 93-117. London: Ubiquity Press. DOI: https://doi.org/10.5334/bbf.e.

Furey, E., Curran, K., Kevitt, P.M., 2013. Probabilistic indoor human movement modeling to aid first responders. Journal of Ambient Intelligence and Humanized Computing 4, 559-569. DOI: https://doi.org/10.1007/s12652012-0112-4

Goodchild, M.F., 2012. The future of Digital Earth. Annals of GIS 18, 93-98. DOI: https://doi.org/10.1080/19475683.2012.668561

Goodchild, M.F., 2007. Citizens as sensors: the world of volunteered geography. GeoJournal 69, 211-221. DOI: https://doi.org/10.1007/s10708-007-9111-y

Goodchild, M.F., Li, L., 2012. Assuring the quality of volunteered geographic information. Spatial Statistics 1, 110-120. DOI: https://doi.org/10.1016/j. spasta.2012.03.002

Goodwin, J., Dolbear, C., Hart, G., 2008. Geographical linked data: The administrative geography of Great Britain on the semantic web. Transactions in GIS 12, 19-30. DOI: https://doi.org/10.1111/j.1467-9671.2008.01133.x

Haklay, M., 2010. How good is volunteered geographical information? A comparative study of OpenStreetMap and Ordnance Survey datasets. Environment and Planning B: Planning and Design 37, 682-703. DOI: https://doi. org/10.1068/b35097

Iliffe, M., 2012. Towards understanding the factors of integrating Spatial Data Infrastructures and crowdsourced geographic data, in: Proceedings of the 1st AGILE PhD School. Wernigerode, Germany, 13-14 March 2012, pp. 71-76. Available at: https://agile-online.org/conference_paper/images/phddocs/ proceedings_agile_phd_school_2012.pdf [Last accessed 16 May 2017].

ISO, 2013. ISO 19157:2013-Geographic information-Data Quality. International Organization for Standardization, Geneva, Switzerland.

Jankowski, P., Andrienko, N., Andrienko, G., Kisilevich, S., 2010. Discovering landmark preferences and movement patterns from photo postings. Transactions in GIS 14, 833-852. DOI: https://doi.org/10.1111/j.14679671.2010.01235.x

Kahila, M., Kyttä, M., 2009. SoftGIS as a bridge-builder in collaborative urban planning, in: Geertman, S., Stillwell, J. (Eds.), Planning Support Systems Best Practice and New Methods. Springer Netherlands, Dordrecht, pp. 389-411.

Kahila-Tani, M., Broberg, A., Kyttä, M., Tyger, T., 2016. Let the citizens mapPublic participation GIS as a planning support system in the Helsinki Master Plan process. Planning Practice \& Research 31, 195-214. DOI: https:// doi.org/10.1080/02697459.2015.1104203

Lin, H., Chen, M., Lu, G., Zhu, Q., Gong, J., You, X., Wen, Y., Xu, B., Hu, M., 2013. Virtual Geographic Environments (VGEs): A new generation of geographic analysis tool. Earth-Science Reviews 126, 74-84. DOI: https://doi. org/10.1016/j.earscirev.2013.08.001 
Longley, P.A., Adnan, M., 2016. Geo-temporal Twitter demographics. International Journal of Geographical Information Science 30, 369-389. DOI: https://doi.org/10.1080/13658816.2015.1089441

Lopez-Pellicer, F., Barrera, J., 2014. Linked Map requirements definition and conceptual architecture, Deliverable D15.1, Available at http://www.planetdata.eu/sites/default/files/PD\%20D15.1.pdf [Last accessed 1 May 2017].

Mangano, D., 2013. The Integrated Data Hub: The Next Generation Data Warehouse. Createspace, S.l.

Massa, P., Campagna, M., 2016. Integrating authoritative and Volunteered Geographic Information for spatial planning, in: Capineri, C., Haklay, M., Huang, H., Antoniou, V., Kettunen, J., Oostermann, F., Purves, R. (Eds.), European Handbook of Crowdsourced Geographic Information. Ubiquity Press, London, UK, pp. 401-418. DOI: https://doi.org/10.5334/bax

Massa, P., Campagna, M., 2014. Social Media Geographic Information: Current developments and opportunities in urban and regional planning, in: Proceedings of the 19th International Conference on Urban Planning and Regional Development in the Information Society. Presented at the GeoMultimedia 2014. DOI: https://doi.org/10.6092/1970-9870/2500

McDougall, K., 2009. Volunteered Geographic Information for building SDI, in: Ostendorf, B., Baldock, P., Bruce, D., Burdett, M., Corcoran, P. (Eds.), Proceedings of the Surveying and Spatial Sciences Institute: Biennial International Conference. Adelaide, Australia, 28 Sep-2 Oct 2009, Surveying and Spatial Sciences Institute, pp. 645-653.

Moens, M.-F., Li, J., Chua, T.-S. (Eds.), 2014. Mining User Generated Content, Social Media and Social Computing. CRC Press, Boca Raton, FL.

Mooney, P, Olteanu-Raimond, A-M, Touya, G, Juul, N, Alvanides, S and Kerle, N. 2017. Considerations of Privacy, Ethics and Legal Issues in Volunteered Geographic Information. In: Foody, G, See, L, Fritz, S, Mooney, P, Olteanu-Raimond, A-M, Fonte, C C and Antoniou, V. (eds.) Mapping and the Citizen Sensor. Pp. 119-135. London: Ubiquity Press. DOI: https://doi. org/10.5334/bbf.f.

Odobašić, D., Medak, D., Miler, M., 2013. Gamification of geographic data collection, in: Thomas, J., Car, A., Strobl, J., Griesebner, G. (Eds.), GI_Forum: Creating the GISociety. Wichman-Verlag, Berlin, pp. 328-337.

Olteanu-Raimond, A.-M., Hart, G., Foody, G., Touya, G., Kellenberger, T., Demetriou, D., 2017. The scale of VGI in map production: A perspective of European National Mapping Agencies. Transactions in GIS 21, 74-90. DOI: https://doi.org/10.1111/tgis.12189

Open Knowledge Foundation, 2013. The Data Hub - The easy way to get, use and share data. Available at http://datahub.io/sq/about [Last accessed 1 May 2017].

Parker, C.J., May, A., Mitchell, V., 2012. Understanding design with VGI using an information relevance framework. Transactions in GIS 16, 545-560. DOI: https://doi.org/10.1111/j.1467-9671.2012.01302.x 
Schade, S., Granell, C., Diaz, L., 2010. Augmenting SDI with Linked Data. Available from: http://ceur-ws.org/Vol-691/paper3.pdf.

Steven, A.R., 2005. The US National Spatial Data Infrastructure: What is new? Presented at the ISPRS Workshop on Service and Application of Spatial Data Infrastructure, Hangzhou, China, 14-16 October 2005. Available at: http://www.isprs.org/proceedings/XXXVI/4-W6/papers/313-318FGDCPaprChina14Oct05.pdf [Last accessed 16 May 2017].

Tang, K.P., Lin, J., Hong, J.I., Siewiorek, D.P., Sadeh, N., 2010. Rethinking location sharing: exploring the implications of social-driven vs. purposedriven location sharing, in: Proceedings of the 12th ACM International Conference on Ubiquitous Computing. ACM Press, New York, NY, USA, pp. 85-94. DOI: https://doi.org/10.1145/1864349.1864363

Wiemann, S., Bernard, L., 2014. Linking crowdsourced observations with INSPIRE, in: Huerta, J., Schade, S., Granell, G. (eds.), Proceedings of the 17th AGILE International Conference on Geographic Information Science: Connecting a Digital Europe through Location and Place, Castellón, Spain, 3-6 June 2014, Available at: https://agile-online.org/conference_paper/cds/ agile_2014/agile2014_80.pdf [Last accessed 16 May 2017].

Wikimapia, 2015. Wikimapia. Available at http://old.wikimapia.org [Last accessed 1 May 2017].

Williamson, I.P., Rajabifard, A., Feeney, M.-E.F. (Eds.), 2003. Developing spatial data infrastructures: from concept to reality. Taylor \& Francis, London; New York. 
